\title{
A Link Communities Analysis of the UK Instagram Covid-19 Searchable Metadiscourse
}

\author{
Chamil Rathnayake \\ University of Strathclyde \\ Chamil.rathnayake@strath.ac.uk
}

\begin{abstract}
Many studies focus on utterances included within specific hashtags. This approach does not adequately acknowledge the fact that social media discourse is distributed across a wide range of hashtags. To assess such 'width of discourse', we examine the extent to which dominant hashtags in the UK Covid-19 Instagram discourse connect with hashtags related to different aspects of social life. A network of Instagram hashtags (31,742 nodes and 107,367 edges) constructed using \#nhsheroes, \#captaintommoore, and \#carehomes as key access points was analysed. Results showed that top nodes in the network representing these and other topics related to the pandemic are highly intertwined. Moreover, results revealed that despite connections among each other, hubs in this network tend to connect more with low degree frames, connecting secondary hashtags to the Covid-19 searchable discourse. Results revealed that a diverse set of local structures consisting of secondary frames are nested within pandemic related communities.
\end{abstract}

\section{Introduction}

Social media hashtags - digital markers used to self-categorise content or develop a thread of conversations [1] - perform a range of functions on Social Network Sites (SNS). They allow organising collective phenomena around focal points, such as social issues (e.g., \#blacklivesmatter), brands (e.g., \#louisvuitton) events (e.g., \#eurovision), political actors (e.g., \#borisjohnson), and public figures (e.g., \#neildegrassetyson). Hashtags serve as social metadata as well as parts of linguistic structure and discourse semantics [2]. Different theoretical perspectives, such as issue publics [3], momentary connectedness [4], brand publics [5], and affective publics [6] conceptualise the role hashtags play in enabling collective phenomena. Such work shows how hashtags serve as spaces for mobilising various user groups via affective as well as discursive forms of engagement. The use of hashtags to mark a given post is an act of indicating relevance of an utterance to a

\author{
Daniel D. Suthers \\ University of Hawai'i at Mānoa \\ Suthers@hawaii.edu
}

specific group of utterances. This is an act of framing, as hashtags serve as technical access points to those groups of utterances. These groups of utterances or frames can be seen as domains where specific aspects of social issues are discussed.

Empirical studies that examine a few distinct hashtags attached to a specific issue are common in social media research (e.g., [7,8]). Focusing on utterances made within specific hashtag frames (in other words, posts that contain a given hashtag) allows analysis of content within those frames, but results in a lack of emphasis on the fact that social media posts are usually marked by a multitude of hashtags. Such hashtags may frame specific local issues (e.g., \#nightingalehospital) as well as more general and internationally used topics (e.g., \#coronavirus). Although there is work that examines the presence of multiple hashtags within specific aspects of social life (e.g., [9], [10]) there is a lack of work that provides a conceptual basis for such inquiry.

This paper emphasizes the need for empirical work that embraces the above mentioned multifaceted nature of social media discourse, especially the range of searchable groups of posts made available to users via multiple hashtags available in posts. We suggest that while the common approach-i.e., analysis of commentary in a single or few given hashtags - allows understanding 'the depth of discourse' within chosen hashtags, analysis of connections among groups of hashtags can demonstrate how specific issue frames are embedded in or contribute to construct a wider searchable discourse. The goal of this study is to examine such 'width of discourse' in Instagram posts related to Covid-19, with a special emphasis on the UK. Our goal is two-fold. From a methodological perspective, we aim to demonstrate, using overlapping community analysis, that social media hashtags should be examined with an emphasis on the extent to which some issue frames, trending hashtags for instance, permeate multiple communities of hashtags. Moreover, we aim to examine how micro level conversations are nested within larger topical communities. The study shows how engagement related to three key domains of the UK public response 
to the Coronavirus - i.e., 1) acknowledgement of the contribution made by keyworkers, 2) widely appreciated fundraising effort by the veteran Captain Sir Thomas Moore, and 3) public dialogue related to increasing fatalities in elder care homes - span across a wide range of topics.

Instagram content related to Covid-19 in the UK is highly diverse and represents different domains of engagement such as responses that are directly tied to the government Coronavirus response (e.g., \#stayalert), work from home (e.g., \#workfromhomeuk), appreciation of the staff of the National Health Service (e.g., \#nhsheroes, \#thankyounhs), and more general reactions (e.g., \#socialdistancing, \#coronavirus). Moreover, there is a wide range of secondary frames, \#portrait, \#londonlife, \#cookingathome, \#food, \#knitting, \#freshbread that are used in conjunction with such dominant hashtags. Based on a network dataset that includes 31,742 hashtags constructed using three key hashtags \#nhsheroes, \#captaintommoore, and \#carehomes - as access points, we examine how primary (dominant) frames mobilise social media discourse. Accordingly, this study asks the following questions:

- To what extent do dominant hashtags connect with secondary frames and permeate different 'discursive communities'?

- What micro level groups of hashtags are nested within broader topical communities?

From the perspective of engagement, answering these questions is important as the three hashtags chosen for analysis represent mobilisation of the public based on valorisation of key workers, charity, and the emphasis on protecting the most vulnerable population.

\section{Hashtags as Issue Frames}

Use of specific hashtags as devices that frame collective phenomena, social and political issues in particular, informs a substantial number of studies that examine their use in different contexts. For instance, Jaramillo-Dent and Pérez-Rodríguez [7] analyse a sample of Instagram posts that contain \#MigrantCaravan and \#CaravanaMigrante to discuss establishment of otherness and representation of immigration as a phenomenon. This approach is important as it allows understanding the depth of engagement within chosen frames. Jaramillo-Dent and Pérez-Rodríguez demonstrate how the border wall proposed for the US-Mexico border has become a central component of hashtagged narratives related to immigration, undermining the voice of the migrant. Rosenbaum's [8] analysis shows that Instagram posts and tweets marked by \#TakeAKnee, \#TakeTheKnee and \#TakeAKneeNFL that emerged in response to American Football player Colin Kaepernick kneeling during the national anthem reflect the construction of American identity.

Use of specific hashtags to study social phenomena is based on the view that they serve as issue frames that capture collective phenomena. Papacharissi [11] notes that hashtags such as \#BlackLivesMatter can be considered as framing devices that allow crowds to transform into publics. Papacharissi argues that these publics, driven by affect, materialise discursively through social network sites. She focuses on the Twitter hashtag \#egypt as a specific instance to examine structures of storytelling within such publics. Bruns and Burgess [3] argue that hashtag communities-user groups that may not necessarily share interests, be aware of, or engage with each other but that share a specific textual attributecan act as issue publics gathered around central interests. These issue publics form ad hoc, perform and dissolve within a short time period. In a similar vein, Rathnayake and Suthers [4] show how momentary connectedness - temporally bounded connectivity via issue-response hashtags-manifests internal diversity in terms of patterns of interaction and user orientations organized around the Twitter hashtag \#NepalEarthquake. These studies' emphasis on specific frames suggests that analysis of utterances made within a given hashtag or several hashtags can offer insight into how collective phenomena form and function.

In general, the common practice in social media research is to choose trending hashtags that have semantic relevance to the issue being investigated. Arguably, this practice relates to the fact that hashtags indicate the 'aboutness' of content [12]. While this approach allows understanding depth of discourse within chosen frames, analysis of utterances within one or a few frames can limit the scope of inquiry in numerous ways. First, a range of parallel and/or rival hashtags that are related to the same issue may emerge within specific social phenomena (e.g, \#BlackLivesMatter and \#AllLivesMatter) and the selection of frames for analysis affects the interpretation of online behaviour. Several scholars have highlighted prevalence of such parallel or rival frames. For instance, Blevins, Lee, McCabe, and Edgerton [13] highlight that ideological, conceptual, as well as more specific hashtags (e.g., places and proper names) are used to discuss social issues, such as the Ferguson shooting incident in 2014. Second, and from a broader viewpoint, hashtags that are not directly relevant to a given issue may play a role within related social media discussions. Analysis of specific 
hashtags does not adequately acknowledge the presence of other hashtags within the same set of posts. Colocation-use of multiple hashtags to mark individual posts - is a common practice and the role played by secondary, tertiary or other markers included in posts is not adequately considered in the above approach. This ignores the value of ambient collectives or publics that are made accessible by a given set of social media posts. Figure 1 shows an Instagram post accessed using the hashtag \#nhsheroes. This post contains hashtags such as \#vegan, \#vegetarian, \#healthyrecipes, \#fitness, and \#fitmeals which act as discursive markers (or issue frames) that represent distinct domains of social media discourse. In this post, the two hashtags related to the pandemic, \#nhs and \#nhsheroes, serve as secondary markers among food and health related markers. Yet, an issue frames perspective does not provide adequate conceptual support to acknowledge their membership in a different public, such as the vegan movement.

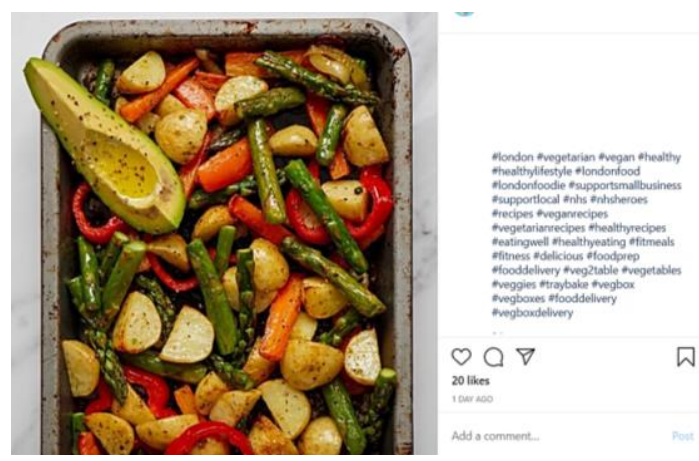

Figure 1: Colocation of Hashtags

Jones [14] notes that the use of multiple hashtags in single posts may not always be semantically meaningful. However, the mere presence of different hashtags is important as they expand the ability of users to navigate multiple publics. There is effort to highlight the importance of mapping co-located hashtags. For instance, Ichau, Frissen, and d'Haenens [9] use hashtag networks to examine themes and representational practices associated with Jewishness and argue that hashtags can carry meanings from other communities. Schöps, Kogler, and Hemetsberger [10] conduct a network analysis of hashtags and show how discursive domains, such as fashion, photography, LGBTQA+ community, and body ideology are embedded in broader structures. Despite such contributions, hashtags remain secondary objects of analysis in social media research. In particular, there is a dearth of work that provides a conceptual basis for analysing co-hashtag networks.

\section{A Relational Approach to Mapping Hashtagged Metadiscourse}

The above argument suggests greater emphasis on hashtags as primary units of analysis and on ambient affiliations. We frame our analysis as an approach to examining the structure of hashtagged metadiscourse. Hyland [15] defines metadiscourse as a term that describes self-reflective expressions that are used to negotiate interactional meanings in a text. This view suggests that metadiscourse assists the writer (or speaker) to express a viewpoint and engage with readers as members of a particular community. Scholars have applied the term to describe the role played by hashtags within and beyond specific utterances, such as tweets. For instance, Zappavigna [2] claims that hashtags function as social media metadiscourse by performing metafunctions including realizing taxonomic classification to enacting metaevaluation as a "conversational aside." She explains that hashtags perform experiential, textual, and interpersonal functions by classifying posts as being of a specific experiential kind, construing relationships by allowing evaluative metacomment, and by organising posts.

Our primary focus is the searchability afforded by hashtags. Zappavigna argues that tagging enables searching social media discourse and supports ambient communion allowed by the ability to find utterances made by others. Zappavigna notes that, from this perspective, connections are ambient as there is sense of presence of others within the network although they may not be necessarily linked to each other via user accounts or conversations. This suggests that hashtags, as social metadata, enables real-time search that allows users to observe how a given social media discourse unfolds. Accordingly, presence of a wide range of markers in a stream of posts means that users are exposed to a wider set of ambient affiliations. These affiliations allow users to navigate different collectives. This argument acknowledges affordances such as navigability [16] that affords user movement through the medium and searchability [17]. We argue that the range of such ambient affiliations made navigable and searchable by hashtags can be seen as the width of social media metadiscourse. Figure 2 captures the essence of our argument. If Hashtag 2 in this figure is chosen as a specific (or primary) issue frame, the stream of posts that includes the hashtag can be examined to understand the depth of discourse within that frame. However, mapping connections among the primary hashtag and other hashtags included in the same stream of posts allows understanding the role secondary, tertiary and other hashtags play in the discourse. We argue that this 
approach allows explaining how different issue publics overlap.

Hashtags are inherently relational digital objects as they link certain posts with other streams of content. From a perspective of discourse, co-occurring hashtags in a given post can be seen as relational elements as the post functions as an interface that connects distinct discourses. Therefore, graph representations of colocated hashtags show the structure of hashtagged metadiscourse that enables searchability of content and navigability between discourses. From the perspective of width of discourse discussed above, co-hashtag networks show the extent to which users can navigate interconnected and overlapping discourses. Table 1 converts the relationships demonstrated in Figure 2 into a graph representation. This conversion is based on the view that co-occurrence connects distinct streams of content by enabling navigation between them. According to the graph given in Table 1, all four hashtags (issue frames) included in Figure 2 can be navigated by accessing any post included in the stream, as posts serve as access points for searchable discourse.

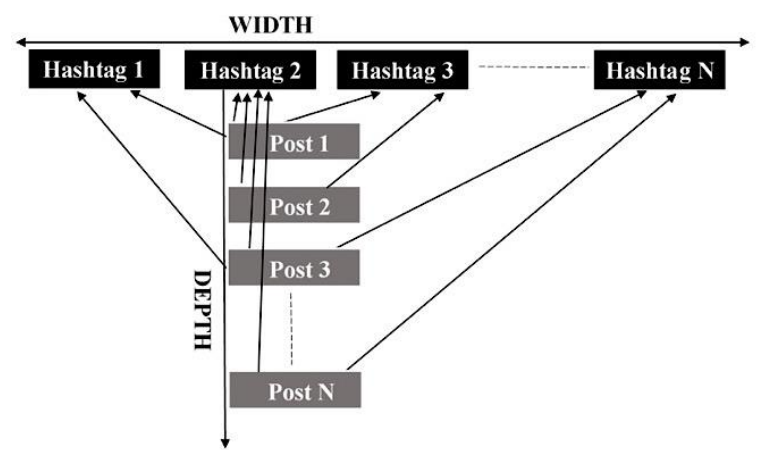

Figure 2: Depth and Width of Social Media Discourse

We make four claims based on the above argument. First, some hashtags will show dominance in terms of degree within a given domain. Second, the extent to which highly connected frames (dominant frames) are connected to less connected frames (dependent frames) shows the ability of dominant frames to mobilise micro level topics. Third, the extent to which specific hashtags belong to different communities of hashtags indicate the ability of certain frames to unify social media discourse. Fourth, the extent to which micro level topical structures are nested in broader structures shows how utterances related to different aspects of the issue overlap. We test these claims empirically by examining a network of Instagram hashtags related to the UK public reaction to the Covid-19 pandemic.
Table 1: Graph Representation of Hashtagged Metadiscourse

\begin{tabular}{|l|ll|}
\hline Post & \multicolumn{3}{l|}{ Relations (Edges) } \\
\hline Post 1 & Hashtag 1 $\Leftrightarrow$ & Hashtag 2 \\
\cline { 2 - 3 } & Hashtag 1 $\Leftrightarrow$ & Hashtag 3 \\
\hline & Hashtag 2 $\Leftrightarrow$ & Hashtag 3 \\
\hline Post 2 & Hashtag 2 $\Leftrightarrow$ & Hashtag 3 \\
\hline Post 3 & Hashtag 1 $\Leftrightarrow$ & Hashtag 2 \\
\hline & Hashtag 1 $\Leftrightarrow$ & Hashtag N \\
\hline & Hashtag 2 $\Leftrightarrow$ & Hashtag N \\
\hline Post N & Hashtag 2 $\Leftrightarrow$ & Hashtag N \\
\hline Graph Representation & \\
\hline
\end{tabular}

\section{Method}

We approach the UK Covid-19 Instagram public discourse using three key frames: \#nhsheroes, \#captaintommoore, and \#carehomes. The hashtag \#nhsheroes emerged as a general public reaction in appreciation of the contribution made by the staff of the UK National Health Service (NHS). The second hashtag (\#captaintommoore) was a more specific frame that emerged as a response to the highly successful fundraising effort by the veteran Tom Moore [18]. Captain Moore raised more than $£ 32$ million to support NHS Charities Together Covid-19 Urgent Appeal by walking 100 laps in his garden on his $100^{\text {th }}$ birthday in exchange for pledges. Both of these hashtags are important to understand the Covid19 public discourse in the UK, especially because they were consistent with the first phase of the government response lead by the slogan "Coronavirus: stay at home, protect the NHS, save lives." The third hashtag (\#carehomes) was used to discuss the issue of high levels of fatalities recorded in care homes in the UK [19]. In the UK, care homes provide residential care for elderly people, such as those who require dementia care, nursing care, and end-of-life care.

A network dataset that included 31,742 hashtags (nodes) and 107,367 co-occurrences (edges) was collected on May 05, 2020. The dataset was collected using the 4CAT data capture tool that allows collecting a maximum of 2500 posts for each main hashtag used as key markers (\#nhsheroes, \#captaintommoore, and \#carehomes). Hashtag networks were extracted from these posts using a R script and were combined to create the final dataset. We use three metrics (degree, degree assortativity, and 
community centrality) to examine the first three claims mentioned above. Degree (the number of edges per node) was calculated to examine the dominance of individual hashtags in the network. Degree assortativity (the extent to which nodes of comparable degree levels connect with each other [20]) was calculated to examine whether dominant frames connect more with each other or with low-degree nodes. We argue that degree assortativity can be used as a measure of whether a given networked metadiscourse has an elite or a grassroots orientation. Hubs link to hubs in assortative networks [20], and in metadiscourse, high assortativity shows an elite structure in which popular frames are associated with each other. Conversely, disassortative structures show a more hierarchical orientation where dominant frames mobilise a range of low-degree frames.

Link community analysis [21], [22] was used to examine the extent to which hashtags permeate different communities. Ahn et al. [21] argue that a fundamental limitation of common methods used for community detection - identification of subgraphs or clusters within a network - is the assumption that any given node can belong to only one community. The link community approach they propose embraces the fact that nodes may belong to multiple communities via different relationships, and communities of nodes can overlap. The method considers similarities between links that share nodes and uses hierarchical clustering to form maximum density partitions of links. This partition induces membership of nodes in (potentially multiple and overlapping) node communities. This fits the fact that a specific hashtag may fit with utterances in completely different domains. For instance, \#nhsheroes has been used in the post given in Figure 1 to annotate a picture of food. Therefore, we can expect \#nhsheroes to be a member of food related social media discourses, as well as others. We perform link community analysis on three levels. First, we visualise the largest communities in the network (i.e, clusters that include the highest number of nodes). Second, community centrality was calculated for all the nodes to calculate the extent to which frames reach out to multiple communities. Finally, we examine topical communities nested within broader clusters of hashtags.

\section{Results and Discussion}

\subsection{Dominant Frames}

Table 2 shows the top ten nodes in the network based on their degree values (i.e., number of other coappearing hashtags). Not surprisingly, the hasthags usd to gather the data (\#carehomes, \#nhsheroes and \#captaintommoore) had high degree values. Other hashtags that had high degree values, such as \#nhs, \#stayhome, \#coronavirus, \#staysafe, \#covid19, and \#clapforourcarers indicate specific and interconnected topics related to the pandemic. In general, these frames indicate a positive stance towards keyworkers. Moreover, \#stayhome directly aligns with the government slogan "Stay at Home, Protect the NHS, Save Lives." The highest edge weights (i.e., number of co-occurrences for each pair of hashtags) given in Table 3 show that \#nhs is used frequently with \#nhsheroes and \#captaintommoore. Moreover, \#captaintommoore and \#nhsheroes occur together frequently in the network. This shows that Instagram engagement related to the veteran's fundraising effort and the general positive reaction towards the NHS are highly intertwined. Moreover, \#nhsheores appeared frequently together with \#stayhome and \#staysafe. From the perspective of metadiscourse, unity in frames and high edge weights between hashtags discussed above show that users envision these frames as related and that they project their utterances to these multiple publics. Moreover, the connected nature of these discursive frames indicates how advice for behavioural control (\#stayhome and \#staysafe) worked with frames that appreciated the health service and keyworkers. This behaviour is consistent with the measures taken by the government to minimise the pressure on NHS as outlined in its strategy [23].

Table 2: High Degree Nodes

\begin{tabular}{|l|l|}
\hline Hashtag & Degree \\
\hline \#carehomes & 4254 \\
\hline \#nhsheroes & 4088 \\
\hline \#nhs & 3367 \\
\hline \#captaintommoore & 2507 \\
\hline \#stayhome & 1457 \\
\hline \#coronavirus & 1168 \\
\hline \#staysafe & 1168 \\
\hline \#covid19 & 1098 \\
\hline \#clapforourcarers & 1072 \\
\hline \#keyworkers & 952 \\
\hline
\end{tabular}

Table 3: Highly Weighted Edges

\begin{tabular}{|lll|l|}
\hline Edge & $\Leftrightarrow$ & \#nhsheroes & Weight \\
\hline \#nhs & 2669 \\
\hline \#captaintommoore & $\Leftrightarrow$ & \#nhs & 1591 \\
\hline \#captaintommoore & $\Leftrightarrow$ & \#happybirthday & 1101 \\
\hline \#clapforourcarers & $\Leftrightarrow$ & \#nhsheroes & 1061 \\
\hline \#captaintommoore & $\Leftrightarrow$ & \#nhsheroes & 1032 \\
\hline \#nhsheroes & $\Leftrightarrow$ & \#stayhome & 977 \\
\hline \#nhs & $\Leftrightarrow$ & \#stayhome & 837 \\
\hline \#captaintommoore & $\Leftrightarrow$ & \#hero & 785 \\
\hline \#carehome & $\Leftrightarrow$ & \#carehomes & 770 \\
\hline \#nhsheroes & $\Leftrightarrow$ & \#staysafe & 696 \\
\hline
\end{tabular}


High edge weights among dominant frames raises the question of whether such frames constitute an elite discourse that does not adequately mobilise a wide range of low degree frames. However, negative degree assortativity (-0.177) indicated that, despite connections among each other, hubs in this network tend to connect more with low degree frames. Arguably, an elite discourse is restrictive as it emphasises popular ambient affiliations, and mainly promotes navigability among those frames. Conversely, a disassortative metadiscourse is more expansive as low degree frames serve as access points to navigate hubs as well as a range of less popular frames. From this perspective, users construct the Covid-19 metadiscourse in a way in which they pair dominant hashtags with a wider range of secondary frames. This can be considered as a mobilising effect as users tie utterances on various topics to trending Covid-19 frames. This also shows the extent to which different domains of social life are tied to the pandemic.

\subsection{Link Communities}

Link communities were computed using the linkcomm package in R 3.6.2, on a $2.8 \mathrm{GHz}$ Intel Core i7 with 16 GB memory and SSD for scratch files. Method getLinkCommunities (hcmethod = "single", directed $=$ FALSE, edglim $=35000$, removetrivial $=$ TRUE) required about 12 hours, and method getAllNestedComm with plot=TRUE required about 6 days to complete.

The link community results showed that the network included 5,012 nontrivial link communities and 514 instances of nesting. While the largest cluster had 466 nodes, there was also a large number of small communities in the network. Figure 3 (next page) visualizes the five largest communities in the network. We notice that \#nhs and \#nhsheroes appear in both partition (a) and (e). Moreover, \#carehomes appears as the top node in two large partitions. In general, these partitions show that dominant frames, such as \#nhs, \#nhsheroes, and \#carehomes do not belong to single communities and that there are multiple communities organised around them.

Table 4 shows high degree nodes in the largest partitions visualised in Figure 3. According to these results, there are overlapping frames between the first and second community, such as \#rescuedog, \#rescuedogsofinstagram and \#royalmarines. This shows that while \#nhsheroes and \#captaintommoore are included in separate communities, there is a substantial level of overlap between the communities lad by these frames. The strong connection between hashtags related to NHS and Captain Tom Moore shows the impact successful charity campaigns play in mobilising public in support of the NHS. From a broad viewpoint, positive connotation reflected by those hashtags is consistent with the favourable attitude towards the NHS that was reported in public satisfaction surveys. For instance, in 2019, the overall public satisfaction with the NHS was at $60 \%$ [24]. The third and fourth communities in Figure 3 are led by \#carehomes. However, these two communities have distinct foci. The third community includes hashtags related to luxury care homes and care home interior design. Moreover, this community includes several internally focused hashtags such as \#usa and \#who. Conversely, the top nodes in Community 4 focus on elderly care. Moreover, this partition has a regional focus: hashtags such as \#sacramento and \#fresno indicate that users specifically connect their locations (in California) with the general hashtag \#carehomes.

From a conceptual perspective, the diversity of the above communities show signs of polymorphismi.e., presence of multiple orientations in issue-response networks [4]. On the other hand, the crucial role played by dominant frames in leading multiple distinct partitions show their ability to unify Instagram metadiscourse by serving as a conduit that connects those communities.

\subsection{Community Centrality and Nested Communities}

Community centrality - a centrality measure that weights the number of communities that a given node belongs to based on how distinct the communities are from each other [22]—was calculated to further examine the extent to which each hashtag is connected to different communities. Table 5 shows community centrality values for hashtags sampled from the distribution at four different intervals (from high to low). The results show that hashtags with high community centrality values are highly relevant to the Covid-19 discourse while hashtags that have low community centrality values are less relevant. This shows that hashtags related to the pandemic reach out to distinct communities. In other words, users in different discursive communities connect hashtags related to the pandemic, such as \#nhs, \#nhsheroes, \#coronavirus, to other domains of engagement. Instagram hashtag \#nhs appears as a dominant frame especially because it was not used as a key hashtag for collecting data. This shows the importance of the NHS to the UK public.

Hashags such as \#surgicalgloves and \#nhsvolunteers are not highly central, but show some community reach. However, general hashtags such as 
\#instamood and \#coconut had no community centrality in the Covid-19 discourse. The connection between community centrality and relevance of hashtags to the pandemic can be seen as a diffusion of Covid-19 discourse to different realms of engagement on Instagram. This allows those who access secondary frames, such as \#food, to navigate the Covid-19 discourse. Positive correlation between degree and community centrality $(0.7414)$ confirms that frequent use of pandemic related hashtags along with a range of other hashtags is highly related to the extent to which dominant hashtags permeate different communities.

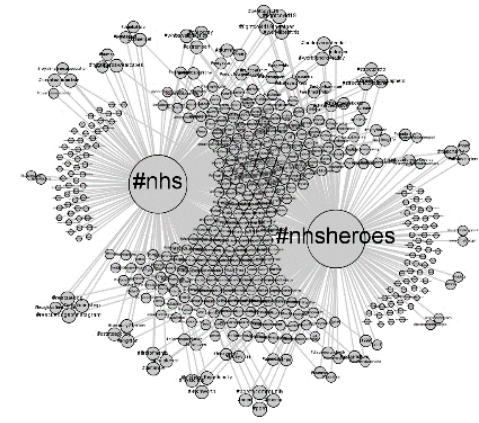

(a) Community 1: 466 nodes, 858 edges

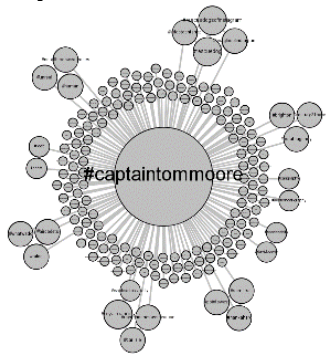

(b) Community 2: 174 nodes, 197 edges

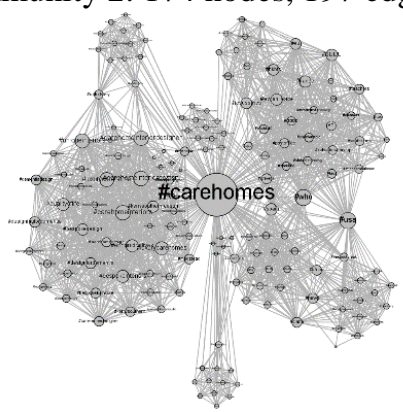

(c) Community 3: 167 nodes, 1940 edges

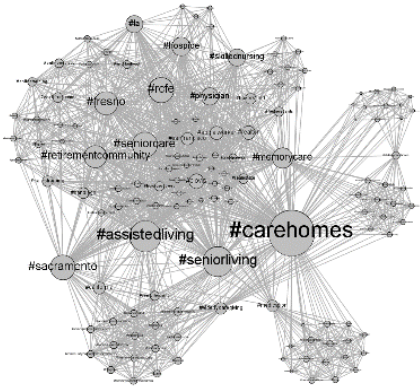

(d) Community 4: 136 nodes, 1594 edges

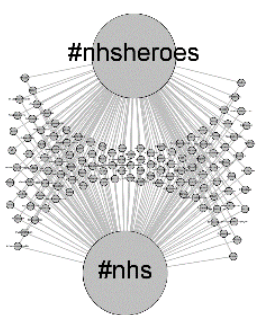

(e) Community 6: 126 nodes, 249 edges

Figure 3: Five Largest Partitions

Table 4: High Degree Nodes in Largest Link Communities

\begin{tabular}{|c|c|}
\hline \multicolumn{2}{|l|}{ Community 1} \\
\hline Hashtag & Degree \\
\hline \#nhsheroes & 401 \\
\hline \#nhs & 391 \\
\hline \#fightcovid19 & 6 \\
\hline \#rescuedog & 6 \\
\hline \#rescuedogsofinstagram & 6 \\
\hline \#poet & 6 \\
\hline \#fightcovid19together & 6 \\
\hline \#poetrycommunity & 6 \\
\hline \#poem & 5 \\
\hline \#royalmarines & 5 \\
\hline \multicolumn{2}{|l|}{ Community 2} \\
\hline Hashtag & Degree \\
\hline \#captaintommoore & 174 \\
\hline \#royalmarines & 5 \\
\hline \#rescuedog & 5 \\
\hline \#rescuedogsofinstagram & 5 \\
\hline \#human & 4 \\
\hline \#adoptdontshop & 4 \\
\hline \#whatwedo & 4 \\
\hline \#aircadets & 4 \\
\hline \#rafac & 4 \\
\hline \#notallheroswearcapes & 4 \\
\hline \multicolumn{2}{|l|}{ Community 3} \\
\hline Hashtag & Degree \\
\hline \#carehomes & 167 \\
\hline \#usa & 60 \\
\hline \#who & 59 \\
\hline \#carehomeinteriordesigner & 54 \\
\hline \#carehomeinteriors & 54 \\
\hline \#carehomeinteriordesign & 54 \\
\hline \#uniqueinteriors & 52 \\
\hline \#luxurycarehome & 52 \\
\hline \#luxurycarehomes & 48 \\
\hline \multicolumn{2}{|l|}{ Community 4} \\
\hline Hashtag & Degree \\
\hline
\end{tabular}




\begin{tabular}{|l|r|}
\hline \#carehomes & 136 \\
\hline \#assistedliving & 93 \\
\hline \#seniorliving & 89 \\
\hline \#seniorcare & 75 \\
\hline \#rcfe & 75 \\
\hline \#sacramento & 74 \\
\hline \#fresno & 70 \\
\hline \#retirementcommunity & 67 \\
\hline \#memorycare & 61 \\
\hline
\end{tabular}

Table 5: Community Centrality Values

\begin{tabular}{|l|l|}
\hline Hashtag & Community Centrality \\
\hline \hline nhsheroes & 318.7319 \\
\hline nhs & 287.2398 \\
\hline carehomes & 230.4797 \\
\hline captaintommoore & 141.7874 \\
\hline coronavirus & 108.1534 \\
\hline \hline nhsvolunteers & 4.273134 \\
\hline surgicalgloves & 4.273134 \\
\hline tissues & 4.273134 \\
\hline messagesofhope & 4.273134 \\
\hline messagesofthanks & 4.273134 \\
\hline \hline captainbritain & 1.153846 \\
\hline bradford & 1.153846 \\
\hline beach & 1.153846 \\
\hline beautifulhuman & 1.153846 \\
\hline artchangeseverything & 1.153846 \\
\hline \hline instamood & 0 \\
\hline wecanallmakeadifference & 0 \\
\hline chichester & 0 \\
\hline coconut & 0 \\
\hline sister & 0 \\
\hline
\end{tabular}

We observed nested communities to examine how secondary topical structures are positioned within communities related to Covid-19 discourse. Figure 4 shows eight selected nested communities. Edges for each community are visualised in different colours. These communities show that a diverse set of local structures consisting of secondary frames, from local hospitals, music, men's fashion and grooming, to animal shows and education, are nested within pandemic related communities. For instance, a local cluster that consists of hashtags on ukulele music (e.g., \#ukuleleband, \#stumming, \#uke) is nested within a community related to the coronavirus (Figure 4a). At the same time, the two main pandemic related hashtags that lead this community (\#covid19 and \#coronavirus) contribute to another community that includes hashtags on elderly care (e.g., \#dementiaactivities, \#elderlyisolation, \#thegoodolddays, 4b). This shows that leading hashtags add an extra community 'layer' in which secondary topics, such as \#ukuleleband, contribute to both a local topical community as well as a broader community that includes Covid-19 hashtags. Similarly, \#nhs and \#nhsheroes contribute to position local topical communities related to areas such as men's fashion and music within broader structures, positioning secondary frames within the Covid-19 metadiscourse (see Figure 4d and Figure 4h). This is an important aspect of user behaviour as connecting secondary frames related to everyday life to the pandemic is crucial in awareness building as well as encouraging behavioural control on a collective level.

The diversity of orientations in topical communities given in Figure 4 indicates polymorphism. However, nesting of those communities within the Covid-19 mestadiscourse complicates the concept. Rathnayake and Suthers [4] argue that polymorphism is present when social media user clusters have different orientations and/or structures. However, in the above nested communities, different orientations co-exist. For instance, in Figure 4(g), the two nested communities have different focii.e., handsanitizers (green edges) and work and recruitment (brown edges). Coexistence of these focuses within a nested community suggests the presence of an internal form of polymorphism.

\section{Conclusion}

The contribution made by this study is twofold. First, we suggest a relational perspective to map Instagram metadiscourse and demonstrate how link community analysis can be used to examine communities within this metadiscourse. Our analysis shows how dominant frames span across a wide range, allowing an assessment of the width of Instagram metadiscourse. Such an analysis can provide useful insight as it allows understanding how users perceived relevance of certain topics to a given digital discourse. Second, and on a more empirical level, the above results show how specific hashtags related to the UK NHS, fundraising effort of the veteran captain, and care homes intersect with each other and with a range of secondary frames. These dominant frames connect different communities unifying the discourse, at least by connecting with less popular frames and allowing seamless navigation among topical communities. The analysis captures the multifaceted nature of Instagram engagement related to Covid-19 in which a range of secondary frames that are not directly related to the pandemic become part of the discourse. This, we argue, emphasizes the role diverse utterances play in understanding how conversations related to social issues are positioned in relation to other conversations. Our work has limitations as only three hashtags were used as access points to gather data. Therefore, a more 


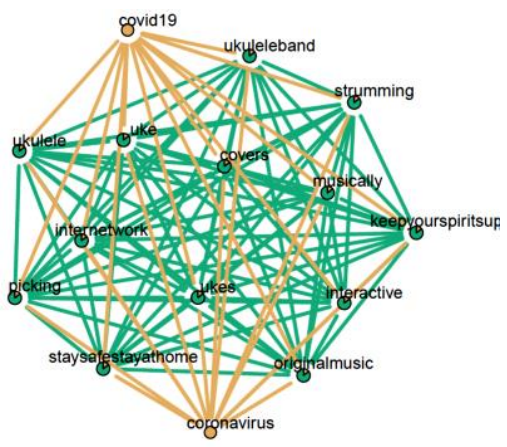

(a) Covid-19/ukulele music

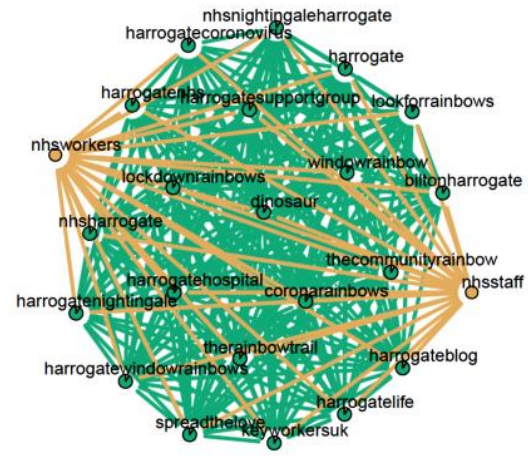

(c) NHS/Harrogate Hospital

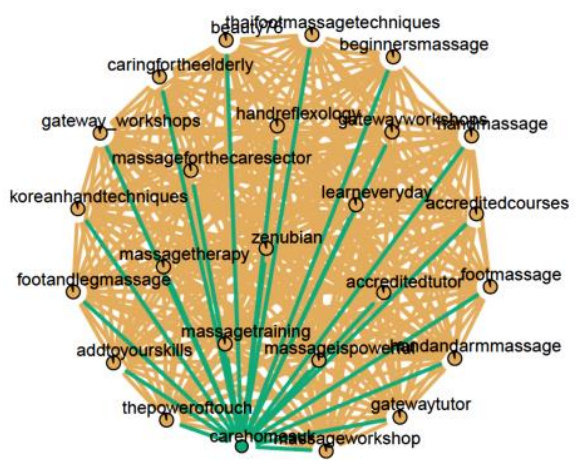

(e) Care homes/Massage therapy and training

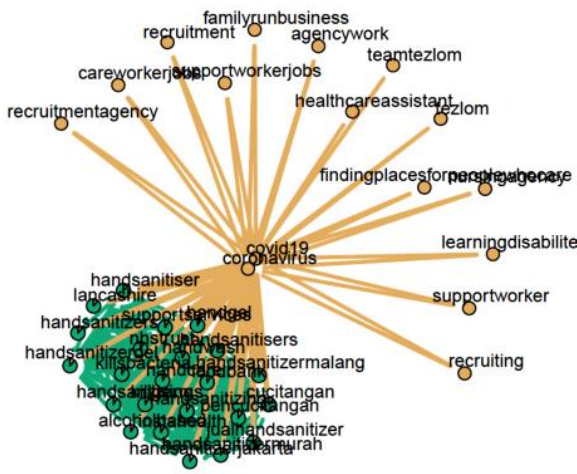

(g) handsanitsers/ work and recruitment

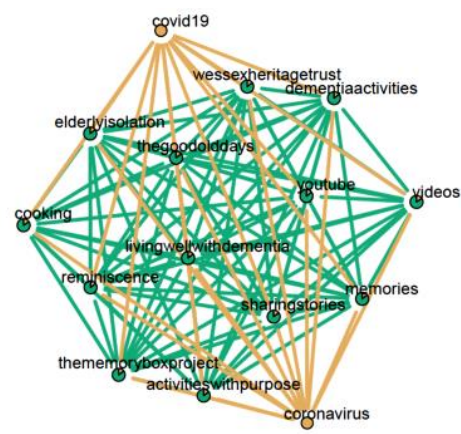

(b) Covid-19/elderly care and socialising

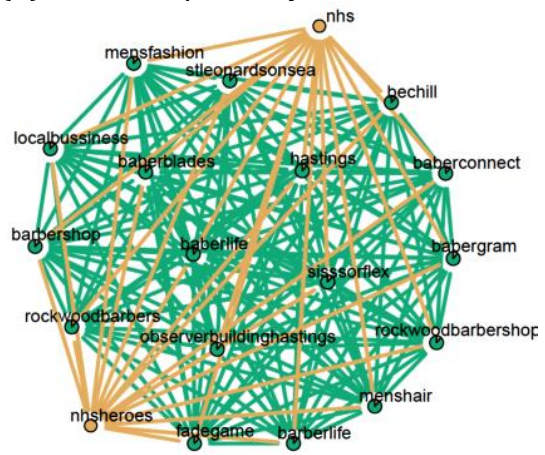

(d) NHS/ mens fashion

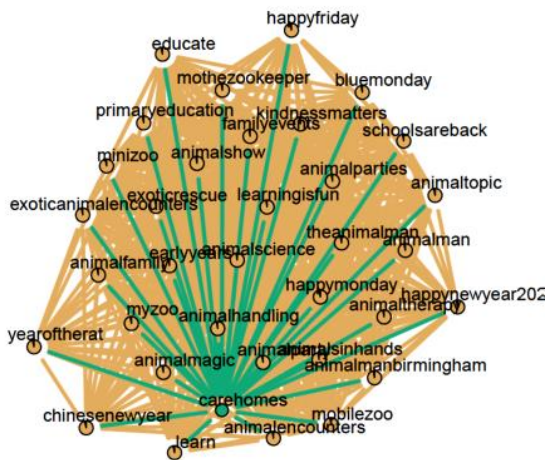

(f) Care homes/ animal shows and education

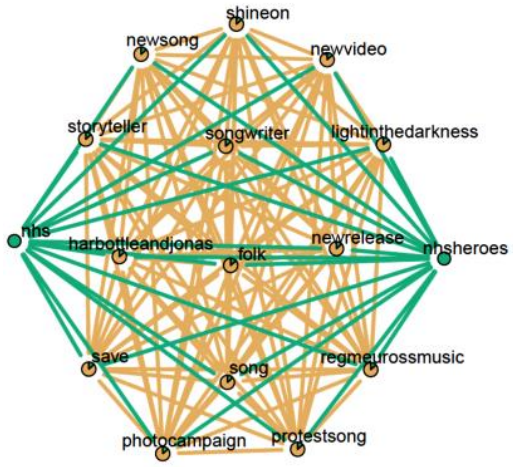

(h) NHS/music

Figure 4: Nested Communities 
comprehensive analysis can be done in order to assess the width of the Covid-19 discourse. A diverse set of access points, such as \#covidiot, \#socialdistancing and even mainstream hashtags such as \#bbc can be used as access points to develop a wider view. Moreover, future work should examine both width and depth of social media discourse in other social and political issues. As mentioned above, our analysis demonstrates how the width of social media metadiscourse can be assessed from a relational perspective. However, this is only one layer of social media discourse. Further work may extend the proposed method to analyse multiple layers of discourse. In particular, a semantic network approach can be used to examine how certain terms mobilise other terms related to a given topic. This can be done on the level of post captions as well as comments made by followers. Such analysis may examine other SNSs, such as Twitter, as this method can be applied to any platforms that provide hashtag functionality.

\section{References}

[1] A. Caliandro, "Digital methods for ethnography: Analytical concepts for ethnographers exploring social media environments," J. Contemp. Ethnogr., vol. 47, no. 5, pp. 551-578, 2018.

[2] M. Zappavigna, "Searchable talk: the linguistic functions of hashtags," Soc. Semiot., vol. 25, no. 3, pp. 274-291, 2015.

[3] A. Bruns and J. Burgess, "The use of Twitter hashtags in the formation of ad hoc publics," in Paper presented at the 6th European Consortium for Political Research General Conference, 2011.

[4] C. Rathnayake and D. D. Suthers, "Twitter issue response hashtags as affordances for momentary connectedness," Soc. Media + Soc., vol. 4, no. 3, p. $205630511878478,2018$.

[5] A. Arvidsson and A. Caliandro, "Brand public," $J$. Consum. Res., vol. 42, no. 5, pp. 727-748, 2015.

[6] Z. Papacharissi, "Affective publics and structures of storytelling: sentiment, events and mediality," Information, Commun. Soc., vol. 4462, no. January, 2015.

[7] D. Jaramillo-Dent and M. A. Pérez-Rodríguez, "\#MigrantCaravan: The border wall and the establishment of otherness on Instagram," New Media Soc., 2019.

[8] J. E. Rosenbaum, "Degrees of freedom: Exploring agency, narratives, and technological affordances in the \#TakeAKnee controversy," Soc. Media + Soc., vol. 5, no. 2, p. 205630511982612, 2019.

[9] E. Ichau, T. Frissen, and L. d'Haenens, "From \#selfie to \#edgy. Hashtag networks and images associated with the hashtag \#jews on Instagram," Telemat. Informatics, vol. 44, no. April, p. 101275, 2019.

[10] J. D. Schöps, S. Kogler, and A. Hemetsberger, "(De)stabilizing the digitized fashion market on Instagram- dynamics of visual performative assemblages," Consum. Mark. Cult., vol. 23, no. 2, pp. 195-213, 2020.

[11] Z. Papacharissi, "Affective publics and structures of storytelling: sentiment, events and mediality," vol. 4462, 2016.

[12] M. Zappavigna, "Enacting identity in microblogging through ambient affiliation," Discourse Commun., vol. 8, no. 2, pp. 209-228, 2014.

[13] J. L. Blevins, J. J. Lee, E. E. McCabe, and E. Edgerton, "Tweeting for social justice in \#Ferguson: Affective discourse in Twitter hashtags," New Media Soc., vol. 21, no. 7, pp. 1636-1653, 2019.

[14] J. Jones, "Switching in Twitter's hashtagged exchanges," J. Bus. Tech. Commun., vol. 28, no. 1, pp. 83-108, 2014.

[15] K. Hyland, Metadiscourse: Exploring interaction in writing. Bloomsbury Academic, 2019.

[16] S. S. Sundar and A. M. Limperos, "Uses and grats 2.0: New gratifications for new media," J. Broadcast. Electron. Media, vol. 57, no. 4, pp. 504-525, 2013.

[17] danah boyd, "Social Network Sites as networked publics: Affordances, dynamics, and implications," in A networked self: Identity, community, and culture on social network sites, Z. Papacharissi, Ed. New York and London: Routledge Taylor \& Francis Group, 2011, pp. 39-58.

[18] "Captain Tom Moore's 100th Birthday Walk for the NHS," 2020. [Online]. Available: https://www.justgiving.com/fundraising/tomswalk. [Accessed: 25-May-2020].

[19] "www.health.org.uk," 2020. [Online]. Available: https://www.health.org.uk/news-and-comment/chartsand-infographics/deaths-from-any-cause-in-carehomes-have-increased. [Accessed: 28-May-2020].

[20] M. Newman, Networks: An introduction. Oxford University Press, 2010.

[21] Y.-Y. Ahn, J. P. Bagrow, and S. Lehmann, "Link communities reveal multiscale complexity in networks," Nature, vol. 466, pp. 761-765, 2010.

[22] A. T. Kalinka and P. Tomancak, "linkcomm: An R package for the generation, visualization, and analysis of link communities in networks of arbitrary size and type," Bioinformatics, vol. 27, no. 14, pp. 2011-2012, 2011.

[23] Www.gov.uk, "Coronavirus: stay at home, protect the NHS, save lives - web version," 2020. [Online]. Available:

https://www.gov.uk/government/publications/coronavi rus-covid-19-information-leaflet/coronavirus-stay-athome-protect-the-nhs-save-lives-web-version. [Accessed: 14-Apr-2020].

[24] A. John, H. Nina, M. David, M. Jessica, S. Laura, and W. Dan, "Public satisfaction with the NHS and social care in 2018: Results from the British Social Attitudes survey," Nuff. Trust King's fund, no. March, pp. 1-32, 2019. 